\title{
Exact renormalization group computation of the optical conductivity of graphene
}

\author{
Alessandro Giuliani ${ }^{1}$ and Vieri Mastropietro ${ }^{2}$ \\ ${ }^{1}$ Università di Roma Tre, L.go S. L. Murialdo 1, IT-00146 Roma, Italy \\ ${ }^{2}$ Università di Roma Tor Vergata, Viale della Ricerca Scientifica IT-00133 Roma, Italy \\ (Received 17 October 2011; revised manuscript received 28 November 2011; published 12 January 2012)
}

\begin{abstract}
The optical conductivity of a system of electrons on the honeycomb lattice interacting through a retarded electromagnetic field is computed by exact renormalization-group (RG) methods. By truncating the exact RG expression at one loop we find that the conductivity has the universal value $\pi / 2$ times the conductivity quantum up to negligible corrections vanishing as a power law in the limit of low frequencies.
\end{abstract}

DOI: 10.1103/PhysRevB.85.045420

PACS number(s): 71.10.Fd, 72.80.Vp, 05.10.Cc, 05.30.Fk

\section{INTRODUCTION}

Among the remarkable properties of graphene, ${ }^{1}$ the optical conductivity is of special interest. Recent experiments ${ }^{2}$ found that the conductivity in monolayer graphene is essentially constant in a wide range of frequencies between the temperature and the bandwidth. The observed value of the conductivity is equal to $\sigma_{0}=\pi e^{2} /(2 h)$, a universal value that only depends on fundamental constants and not on the material parameters such as the Fermi velocity. This fact is in good agreement with an analytical derivation of the conductivity in a system of noninteracting fermions on the honeycomb lattice at zero chemical potential. ${ }^{3}$ The computation of Ref. 3 extended and confirmed earlier analyses based on the standard effective description of graphene in terms of noninteracting massless Dirac fermions. ${ }^{4-7}$

The remarkable agreement between the measurements in Ref. 2 and a theoretical value computed by neglecting many-body interaction is, however, surprising and needs an explanation. ${ }^{8}$ Indeed, the strength of the interactions in graphene is measured by the ratio $\alpha=\frac{e^{2}}{\hbar v_{0}} \sim 2.2$ ( $e$ is the electric charge and $v_{0}$ is the Fermi velocity), which is 300 times larger than the usual fine-structure constant. The effects of the interactions are clearly seen in experiments on the Fermi velocity. ${ }^{9}$ Therefore, why is there not an essential many-body renormalization of the optical conductivity, too?

On the theoretical side, a theorem proved in Ref. 10 establishes that the conductivity of electrons hopping on the honeycomb lattice and interacting via a weak finite range interaction (e.g., a local Hubbard or a nearest-neighbor interaction) is equal to $\sigma_{0}$ in the limit of zero frequency. Note that, even if dimensionally irrelevant, the interaction can produce finite many-body renormalizations: for instance, the Fermi velocity is renormalized by the interaction. Therefore, the universality of the conductivity is a nontrivial statement, following from an exact cancellation of all the many-body corrections.

It is, however, believed that the interaction in clean suspended graphene is not at all short ranged, so that a realistic description of the clean system requires the inclusion of the long-ranged electromagnetic (e.m.) interactions. In the case of static Coulomb interactions, Ref. 11 predicted a logarithmic renormalization of the Fermi velocity, namely, $v(q)=v_{0}(1+$ $\frac{\alpha}{4} \log \frac{\varepsilon}{q}$ ), where $q$ is the momentum measured from the Fermi points and $\varepsilon$ is the bandwidth. First attempts to include the effects of a Coulomb potential on the conductivity ${ }^{12}$ led to the conclusion that the interaction radically changes its behavior, that is, $\lim _{\omega \rightarrow 0} \sigma(\omega)=0$, where $\sigma(\omega)$ is the conductivity at frequency $\omega$ in the limit of zero temperature. Later, Refs. 13 and 14 obtained the qualitatively different result $\lim _{\omega \rightarrow 0} \sigma(\omega)=\sigma_{0}$, based on scaling arguments. In particular, Ref. 13 found the formula

$$
\sigma(\omega)=\sigma_{0}\left[1+O\left(\frac{1}{\log (\varepsilon / \omega)}\right)\right] .
$$

Note that the inverse logarithmic correction in Eq. (1) is a consequence of the logarithmic divergence of the Fermi velocity and should be read as $O\left[\alpha v_{0} / v(\omega)\right]$. As pointed out in Ref. 8, this correction is in general larger than the experimental error. ${ }^{2}$ References 15 and 16 proposed that the way out from this apparent contradiction should be found in the constant in front of the inverse logarithmic corrections, whose correct value should be much smaller than the one computed in Ref. 13. However, Ref. 17 raised objections against the new value proposed in Refs. 15 and 16, because the regularizations used in these works can produce unphysical results. The disagreement between the big (inverse logarithmic) corrections to the conductivity and the experimental data suggested $^{8}$ to phenomenologically postulate a Fermi-liquid description of the interacting system: this assumption implies that the universal conductivity is reached at low frequencies polynomially fast (i.e., as $\sim \omega^{2}$ ) but is in contrast with the experiments in Ref. 9.

Equation (1) was derived by assuming that the electrons interact via a static Coulomb interaction: however, the logarithmic unbounded increase of the Fermi velocity shows that the assumption of instantaneous interactions becomes unphysical at very low energy scales. ${ }^{18}$ Therefore, the use of Eq. (1) and of the divergence of the Fermi velocity to predict the universality of the conductivity as $\omega \rightarrow 0$ at zero temperature is questionable.

In this paper, we study the graphene's conductivity in the presence of Coulomb interactions, by also taking into account the retardation effects of the e.m. fields; this helps in avoiding some unphysical behaviors, such as the divergence of the renormalized Fermi velocity in the infrared (see Refs. 19 and 20). A lattice gauge model for graphene with retarded e.m. interactions was introduced in Ref. 20 and 
exact renormalization-group ( $\mathrm{RG}$ ) methods allowed us to write the two-point and several response functions in terms of a renormalized series in the effective dressed couplings: the prediction is that the system exhibits a line of infrared fixed points and the correlation functions acquire anomalous critical exponents. There is a deep difference between the perturbative series in the bare coupling and the renormalized one: while the first is plagued by $O\left(\log ^{n} \omega\right)$ divergences at order $n$, the second is order by order finite because the apparent logarithmic divergences are resummed into nontrivial critical exponents. Therefore, its truncation is expected to give much more accurate predictions. A similar renormalized series is written here for the conductivity $\sigma(i \omega)$ in the zero-temperature limit; by truncating it at one loop (of course, the dressed one-loop contribution still corresponds to a resummation of infinitely many diagrams of the nonrenormalized expansion) and by taking $i \omega \rightarrow \omega+i 0^{+}$, we find

$$
\operatorname{Re} \sigma(\omega)=\sigma_{0}\left[1+O\left(\frac{\omega^{2}}{\varepsilon^{2}}\right)\right]
$$

while the imaginary part of the conductivity vanishes at zero frequency linearly in $\omega$. Equation (2) says that in the "collisionless regime," ${ }^{21}$ that is, for $k_{B} T \ll \omega \ll \varepsilon$, the conductivity is very close to the universal one $\sigma_{0}$, up to $a$ really negligible power-law correction, compatible with the experimental results in Ref. 2 and much smaller than the $O\left(\frac{1}{\log (\varepsilon / \omega)}\right)$ correction in Eq. (1). In other words, by taking into account the retarded e.m. field, we obtain results in qualitative agreement with the observed dramatic increase of the Fermi velocity and with the universality of the conductivity up to negligible power-law corrections at low frequencies. Even though the system with long-range interactions belongs to a nontrivial infrared universality class (different from noninteracting massless Dirac fermions) and the correlation functions exhibit anomalous critical exponents, ${ }^{20}$ the lowfrequency behavior of the conductivity is the same as the free one, thanks to dramatic cancellations due to lattice gauge invariance.

The rest of the paper is organized as follows. We first introduce the model, define the conductivity, and explain how to compute it in terms of correlation functions. The latter can be expressed as functional derivatives of the generating functional, which can be analyzed by exact RG methods and can be used to derive exact Ward identities (WIs) between the response functions. Next we sketch the RG analysis and the use of WIs in controlling the flow of the running coupling constants. Finally we specialize the general construction to the computation of the conductivity, getting an explicit formula for it [Eq. (20)], which is investigated in detail in the Appendix.

\section{THE MODEL, THE CONDUCTIVITY AND THE RESPONSE FUNCTIONS}

We describe graphene by a system of fermions on the honeycomb lattice interacting through an e.m. field. The model we consider was defined in detail in Ref. 20. Let us just remind here the main definitions. We let $\Lambda=\left\{n_{1} \vec{l}_{1}+n_{2} \vec{l}_{2}\right.$ : $\left.n_{i}=0, \ldots, L-1\right\}$ be a periodic triangular lattice of period $L$, with basis vectors $\vec{l}_{1}=\frac{1}{2}(3, \sqrt{3}), \vec{l}_{2}=\frac{1}{2}(3,-\sqrt{3})$. We denote by $\Lambda_{A}=\Lambda$ and $\Lambda_{B}=\Lambda+\vec{\delta}_{i}$ the $A$ - and $B$ - sublattices of the honeycomb lattice of mesh 1 , with $\vec{\delta}_{i}$ the nearest neighbors vectors defined as: $\vec{\delta}_{1}=(1,0), \vec{\delta}_{2}=\frac{1}{2}(-1, \sqrt{3})$, $\vec{\delta}_{3}=\frac{1}{2}(-1,-\sqrt{3})$. The grand-canonical Hamiltonian at halffilling is $H=H_{0}+H_{C}+H_{A}$, where

$$
\begin{aligned}
H_{0}= & -t \sum_{\substack{\vec{x} \in \Lambda_{A} \\
j=1,2,3}} \sum_{\sigma=\uparrow \downarrow} a_{\vec{x}, \sigma}^{+} b_{\vec{x}+\vec{\delta}_{j}, \sigma}^{-} \\
& \times \exp \left[i e \int_{0}^{1} \vec{\delta}_{j} \cdot \vec{A}\left(\vec{x}+s \vec{\delta}_{j}, 0\right) d s\right]+\text { c.c. }
\end{aligned}
$$

is the gauge-invariant nearest-neighbor hopping term (here $t$ is the hopping strength, and $a^{ \pm}, b^{ \pm}$are the creation/annihilation operators of electrons sitting at the sites of $\Lambda_{A}$ or $\Lambda_{B}$ ), and

$$
H_{C}=\frac{e^{2}}{2} \sum_{\vec{x}, \vec{y} \in \Lambda_{A} \cup \Lambda_{B}}\left(n_{\vec{x}}-1\right) \varphi(\vec{x}-\vec{y})\left(n_{\vec{y}}-1\right),
$$

where $e$ is the electric charge, $\hat{\varphi}_{\vec{p}}$ is an ultraviolet regularized version of the static Coulomb potential, and $n_{\vec{x}}$ is the electron number at site $\vec{x}$. Finally, $H_{A}$ is the energy (in the presence of an ultraviolet cutoff) of the three-dimensional photon field $\underline{A}=\left(\vec{A}, A^{3}\right)$ in the Coulomb gauge. Units are fixed in such a way that the speed of light $c=1$. Note that the interaction with the quantum e.m. field is introduced via the Peierls substitution in order to preserve gauge invariance.

We define a "space-time" three-component vector $\hat{J}_{\vec{p}, \mu}$, $\mu=0,1,2$, with

$\hat{J}_{\vec{p}, 0}=\exp \left[\sum_{\substack{\vec{x} \in \Lambda_{A} \\ \sigma=\uparrow \downarrow}} e^{-i \vec{p} \vec{x}} a_{\vec{x}, \sigma}^{+} a_{\vec{x}, \sigma}^{-}+\sum_{\substack{\vec{x} \in \Lambda_{B} \\ \sigma=\uparrow \downarrow}} e^{-i \vec{p} \vec{x}} b_{\vec{x}, \sigma}^{+} b_{\vec{x}, \sigma}^{-}\right]$,

the density operator and $\hat{J}_{\vec{p}, 1}, \hat{J}_{\vec{p}, 2}$ the two components of the paramagnetic current

$$
\vec{J}_{\vec{p}}=i e t \sum_{\substack{\vec{x} \in \Lambda \\ \sigma, j}} e^{-i \vec{p} \vec{x}} \vec{\delta}_{j} \eta_{\vec{p}}^{j}\left(a_{\vec{x}, \sigma}^{+} b_{\vec{x}+\vec{\delta}_{j}, \sigma}^{-}-b_{\vec{x}+\vec{\delta}_{j}, \sigma}^{+} a_{\vec{x}, \sigma}^{-}\right),
$$

where $\eta_{\vec{p}}^{j}=\left(1-e^{-i \vec{p} \vec{\delta}_{j}}\right) /\left(i \vec{p} \vec{\delta}_{j}\right)$. Let also $\mathbf{p}=(\omega, \vec{p})$, with $\omega$ the Matsubara frequency, and $\hat{K}_{\mu \nu}(\mathbf{p})$ is the currentcurrent response function, i.e., the Fourier transform of $\lim _{\beta \rightarrow \infty}\left\langle J_{\mathbf{x}, \mu} ; J_{\mathbf{y}, \nu}\right\rangle_{\beta}$.

We are interested in the conductivity, defined via the Kubo formula as ${ }^{3,10}$ (here $l, m=1,2$ )

$$
\sigma_{l m}(i \omega)=-\frac{2}{3 \sqrt{3}} \frac{1}{\omega}\left[\hat{K}_{l m}(\omega, \overrightarrow{0})+\hat{\Delta}_{l m}(\overrightarrow{0})\right],
$$

where $3 \sqrt{3} / 2$ is the area of the hexagonal cell of the honeycomb lattice and

$$
\hat{\Delta}_{l m}(\vec{p})=\lim _{\beta, L \rightarrow \infty} \frac{1}{L^{2}} \sum_{\substack{\vec{x} \in \Lambda \\ j=1,2,3}}\left(\vec{\delta}_{j}\right)_{l}\left(\vec{\delta}_{j}\right)_{m}\left|\eta_{\vec{p}}^{j}\right|^{2}\left\langle\Delta_{\vec{x}, j}\right\rangle_{\beta},
$$

with $\Delta_{\vec{x}, j}=-e^{2} t \sum_{\sigma}\left(a_{\vec{x}, \sigma}^{+} b_{\vec{x}+\vec{\delta}_{j}, \sigma}^{-}+b_{\vec{x}+\vec{\delta}_{j}, \sigma}^{+} a_{\vec{x}, \sigma}^{-}\right)$the diamagnetic tensor. 
The response functions can be computed via the generating functional that, in the Feynman gauge, reads

$$
e^{\mathcal{W}_{h^{*}}(J, \lambda)}=\int P(d \psi) \int P_{h^{*}}(d A) e^{\mathcal{V}(A+J, \psi)+(\psi, \lambda)},
$$

which has been studied in great detail in Ref. 20. In Eq. (4), (i) $\psi_{\mathbf{k}, \sigma}^{ \pm}$are Grassman spinors [of the form $\psi=(a, b)$, with $a$ and $b$ the electron fields associated to the two sublattices of the honeycomb net] and $P(d \psi)$ is the fermionic Gaussian integration with propagator

$$
g(\mathbf{k})=-\frac{1}{Z_{0}}\left(\begin{array}{lc}
i k_{0} & v_{0} \Omega^{*}(\vec{k}) \\
v_{0} \Omega(\vec{k}) & i k_{0}
\end{array}\right)^{-1},
$$

where $Z_{0}=1$ is the free wave-function renormalization, $v_{0}=$ $\frac{3}{2} t$ is the free Fermi velocity, and $\Omega(\vec{k})=\frac{2}{3} \sum_{j=1,2,3} e^{i \vec{k}\left(\vec{\delta}_{j}-\vec{\delta}_{1}\right)}$ is the complex dispersion relation. Note that $g(\mathbf{k})$ is singular only at the Fermi points $\mathbf{p}_{F}^{r}=\left(0, \frac{2 \pi}{3}, r \frac{2 \pi}{3 \sqrt{3}}\right)$, where $r= \pm$ is the valley index. Moreover, $A_{\mu, \mathbf{p}}, \mu=0,1,2$, are the Fourier transform of real Gaussian variables and $P_{h^{*}}(d A)$ is the Gaussian integration with propagator $w_{\mu \nu}(\mathbf{p})=\delta_{\mu \nu}(2|\mathbf{p}|)^{-1} \chi_{\left[h^{*}, 0\right]}(|\vec{p}|)$, where $\chi_{\left[h^{*}, 0\right]}$ is a smooth compact support function that acts both as an ultraviolet cutoff on scale $|\mathbf{p}| \sim 1$ and as an infrared cutoff on scale $|\mathbf{p}| \sim 2^{h^{*}}$ (to be eventually removed). Finally $\mathcal{V}$ is the interaction whose explicit form can be easily inherited from $H .^{20}$ The field-field, the field-current, and the current-current correlations can be obtained by taking the limit $h^{*} \rightarrow-\infty$ of the following functional derivatives of the generating functional:

$$
\begin{aligned}
\hat{S}_{2}^{h^{*}}(\mathbf{k}) & =\left.\frac{\delta^{2} \mathcal{W}_{h^{*}}(J, \lambda)}{\delta \hat{\lambda}_{\mathbf{k}}^{-} \delta \hat{\lambda}_{\mathbf{k}}^{+}}\right|_{J=\lambda=0}, \\
\hat{S}_{2,1 ; \mu}^{h^{*}}(\mathbf{k}, \mathbf{p}) & =\left.\frac{\delta^{3} \mathcal{W}_{h^{*}}(J, \lambda)}{\delta \hat{J}_{\mu, \mathbf{p}} \delta \hat{\lambda}_{\mathbf{k}}^{-} \delta \hat{\lambda}_{\mathbf{k}+\mathbf{p}}^{+}}\right|_{J=\lambda=0}, \\
\hat{K}_{\mu \nu}^{h^{*}}(\mathbf{p}) & =\left.\frac{\delta^{2} \mathcal{W}_{h^{*}}(J, \lambda)}{\delta \hat{J}_{\mu, \mathbf{p}} \delta \hat{J}_{\nu,-\mathbf{p}}}\right|_{J=\lambda=0}-\hat{\Delta}_{\mu \nu}(\mathbf{p}),
\end{aligned}
$$

where in the last line $\hat{\Delta}_{\mu v}(\mathbf{p})$ should be identified with $\sum_{i, j=1,2} \delta_{\mu, i} \delta_{v, j} \hat{\Delta}_{i j}(\vec{p})$.

In writing the generating functional as in Eq. (4) we exploited gauge invariance and, more precisely, the equivalence between the Feynman and the Coulomb gauges. Another crucial consequence of gauge invariance is the following equation:

$$
0=\left.\frac{\partial}{\partial \hat{\alpha}_{\mathbf{p}}} \mathcal{W}_{h^{*}}\left(\Phi, J+\partial \alpha, \lambda e^{i e \alpha}\right)\right|_{\hat{\alpha}=0} .
$$

By performing derivatives with respect to the external fields, Eq. (7) implies a sequence of exact lattice Ward identities, valid for each finite choice of the cutoff scale $h^{*}$. In particular, if $p^{0}=-i \omega, p^{1}=p_{1}$, and $p^{2}=p_{2}$, we get

$$
\begin{gathered}
p^{\mu} \hat{S}_{2,1 ; \mu}^{h^{*}}(\mathbf{k}, \mathbf{p})=e \Gamma(\vec{p}) \hat{S}_{2,0}^{h^{*}}(\mathbf{k})-e \hat{S}_{2,0}^{h^{*}}(\mathbf{k}+\mathbf{p}) \Gamma(\vec{p}), \\
p^{\mu} \hat{K}_{\mu \nu}^{h^{*}}(\mathbf{p})=-p^{\mu} \hat{\Delta}_{\mu \nu}(\mathbf{p})
\end{gathered}
$$

where $\Gamma(\vec{p})=\left(\begin{array}{cc}1 & 0 \\ 0 & e^{-i \vec{p} \vec{p}_{1}}\end{array}\right)$ and summation over repeated indices is understood. Taking the limit $h^{*} \rightarrow-\infty$ in Eq. (9) and using the continuity of $\hat{K}_{\mu \nu}(\mathbf{p})$ in $\mathbf{p}=\mathbf{0}$ (proved at all orders of renormalized perturbation theory in Ref. 20), we find, for $i, j \in$ $\{1,2\}$,

$$
\sigma_{i j}(\omega)=-\frac{2}{3 \sqrt{3}} \frac{\hat{K}_{i j}(\omega, 0)-\hat{K}_{i j}(0, \overrightarrow{0})}{\omega}
$$

where $\hat{K}_{i j}(\omega, 0)$ can be computed from $\mathcal{W}_{h^{*}}$, using Eq. (6). A perturbation theory in $e$ for $\mathcal{W}_{h^{*}}$ is plagued by logarithmic divergences, which make it difficult to extract physical information from the analysis of lowest orders. The generating functional can, instead, be analyzed by exact RG methods, ${ }^{20}$ which allow us to systematically resum the logarithmically divergent contributions in the naive perturbation theory and to recast it into a better behaved expansion, which is finite at all orders.

\section{RG ANALYSIS}

We decompose the fermionic and bosonic fields $\psi_{\mathbf{k}, \sigma}, A_{\mu, \mathbf{p}}$ as sums of fields $\psi_{\mathbf{k}, \sigma, r}^{(h)}, A_{\mu, \mathbf{p}}^{(h)}, h \leqslant 0$, living on momentum scales closer and closer to the singularity of the the propagator; namely, $\psi_{\mathbf{k}, \sigma, r}^{(h)}$ is supported on momenta at a distance $2^{h}$ from $\mathbf{p}_{F}^{r}$, while $A_{\mu, p p}^{(h)}$ is supported on momenta at a distance $2^{h}$ from the origin. The analysis proceeds inductively: after the integration of the fields $\left(\psi^{(0)}, A^{(0)}\right), \ldots,\left(\psi^{(h+1)}, A^{(h+1)}\right)$, we get (setting for notational simplicity $\lambda=0$ )

$e^{\mathcal{W}_{h^{*}}(J, 0)}=\int \prod_{r= \pm} P\left(d \psi_{r}^{(\leqslant h)}\right) P\left(d A^{(\leqslant h)}\right) e^{\mathcal{V}^{(h)}\left(\sqrt{Z_{h}} \psi^{(\leqslant h)}, A^{(\leqslant h)}+J\right)}$,

where $P\left(d \psi_{r}^{(\leqslant h)}\right)$ and $P\left(d A^{(\leqslant h)}\right)$ have propagators

$\hat{g}_{r}^{(\leqslant h)}\left(\mathbf{k}^{\prime}\right)=-\frac{\chi_{h}\left(\left|\vec{k}^{\prime}\right|\right)}{Z_{h}}\left(\begin{array}{cc}i k_{0} & v_{h} \Omega^{*}\left(\vec{k}^{\prime}+\vec{p}_{F}^{r}\right) \\ v_{h} \Omega\left(\vec{k}^{\prime}+\vec{p}_{F}^{r}\right) & i k_{0}\end{array}\right)^{-1}$

and $w_{\mu \nu}^{(\leqslant h)}(\mathbf{p})=\delta_{\mu \nu}(2|\mathbf{p}|)^{-1} \chi_{\left[h^{*}, h\right]}(\vec{p})$, where: (i) $\chi_{h}\left(\vec{k}^{\prime}\right)$ is a smooth cutoff function vanishing for momenta larger than $\left|\vec{k}^{\prime}\right| \sim 2^{h}$; (ii) $\chi_{\left[h^{*}, h\right]}(\vec{p})=\chi_{h}(\vec{p})-\chi_{h^{*}}(\vec{p})$; and (iii) $Z_{h}, v_{h}$ are the effective wave function renormalization and Fermi velocity at scale $h$. Moreover $\mathcal{V}^{(h)}$ is the effective potential, expressed by a sum of monomials in $\psi^{(\leqslant h)}, A^{(\leqslant h)}$ of any degree:

$$
\begin{aligned}
\mathcal{V}^{(h)} & \left(\sqrt{Z_{h}} \psi^{(\leqslant h)}, A^{(\leqslant h)}\right) \\
= & \int \frac{d \mathbf{p}}{(2 \pi)^{3}}\left[Z_{h}^{(\mu)} e \hat{J}_{\mu, \mathbf{p}}^{(\leqslant h)} \hat{A}_{\mu, \mathbf{p}}^{(\leqslant h)}-2^{h} v_{\mu, h} \hat{A}_{\mu,-\mathbf{p}}^{(\leqslant h)} \hat{A}_{\mu, \mathbf{p}}^{(\leqslant h)}\right] \\
& +\mathcal{R V}^{(h)}\left(\sqrt{Z_{h}} \psi^{(\leqslant h)}, A^{(\leqslant h)}\right),
\end{aligned}
$$

where $\mathcal{R V}^{(h)}\left(\sqrt{Z_{h}} \psi^{(\leqslant h)}, A^{(\leqslant h)}\right)$ is the irrelevant part of the effective potential (sum of all the terms with more than three fields) and

$$
\hat{J}_{\mu, \mathbf{p}}^{(\leqslant h)}:=\frac{i}{\beta L^{2}} \sum_{r, \sigma, \mathbf{k}^{\prime}} \hat{\psi}_{\mathbf{k}^{\prime}+\mathbf{p}, \sigma, r}^{(\leqslant h)+} \Gamma_{r}^{\mu}\left(\vec{k}^{\prime}\right) \hat{\psi}_{\mathbf{k}^{\prime}, \sigma, r}^{(\leqslant h)-},
$$

with $\Gamma_{r}^{0}(\vec{k})=\mathbb{1}$ and

$$
\Gamma_{r}^{i}\left(\vec{k}^{\prime}\right)=\frac{2}{3} \sum_{j=1}^{3}\left(\vec{\delta}_{j}\right)_{i}\left(\begin{array}{cc}
0 & i e^{-i\left(\vec{k}^{\prime}+\vec{p}_{F}^{r}\right)\left(\vec{\delta}_{j}-\vec{\delta}_{1}\right)} \\
-i e^{i\left(\vec{k}^{\prime}+\vec{p}_{F}^{r}\right)\left(\vec{\delta}_{j}-\vec{\delta}_{1}\right)} & 0
\end{array}\right) .
$$


Using the properties of the Gaussian fermionic integration, we can write in Eq. (11),

$$
P\left(d \psi_{r}^{(\leqslant h)}\right)=P\left(d \psi_{r}^{(\leqslant h-1)}\right) P\left(d \psi_{r}^{(h)}\right),
$$

where $P\left(d \psi_{r}^{(h)}\right)$ is the "single scale" integration with propagator $g_{r}^{(h)}\left(\mathbf{k}^{\prime}\right)$, which is given by the same expression as Eq. (12) with $\chi_{h}\left(\left|\vec{k}^{\prime}\right|\right)$ replaced by the smooth compact support function $f_{h}\left(\left|\vec{k}^{\prime}\right|\right):=\chi_{h}\left(\left|\vec{k}^{\prime}\right|\right)-\chi_{h-1}\left(\left|\vec{k}^{\prime}\right|\right)$; note that $f_{h}\left(\left|\vec{k}^{\prime}\right|\right)$ is nonvanishing only if $\vec{k}^{\prime}$ is on scale $h$, i.e., $2^{h-1} \leqslant\left|\vec{k}^{\prime}\right| \leqslant 2^{h+1}$. Similarly we can write $P\left(d A^{(\leqslant h)}\right)=P\left(d A^{(\leqslant h-1)}\right) P\left(d A^{(h)}\right)$. By integrating Eq. (11) with respect to $\psi^{(h)}, A^{(h)}$, we get an expression that can be recast in the same form, with $h-1$ replacing $h$, so that the procedure can be iterated.

Equation (11) can be physically read by saying that, as a consequence of the renormalizability of the model, the effect of the interaction is simply to produce a renormalization of the effective parameters, namely, the Fermi velocity $v_{h}$, the wave function renormalization $Z_{h}$, the vertex function $Z_{h}^{(\mu)}$, and the photon mass $v_{\mu, h}$. The discrete rotational symmetries of the model imply that $Z_{h}^{(1)}=Z_{h}^{(2)}$ and $\nu_{1, h}=v_{2, h}$. The inductive integration procedure induces a renormalized expansion for $\mathcal{W}_{h^{*}}$ in terms of the effective charges $e_{0, h}=e Z_{h}^{(0)} / Z_{h}$ and $e_{i, h}=e Z_{h}^{(i)} /\left(Z_{h} v_{h}\right), i \in\{1,2\}$, which is meaningful only if $e_{\mu, h}$ remain small for all the $\mathrm{RG}$ steps.

The smallness of the effective charges can be proved by making use of gauge invariance. The key idea is to use the WIs, Eqs. (8) and (9) with a finite bosonic cutoff and to note that the quantities involved in such WIs depend on the effective parameters at scale $h^{*}$; indeed, for $\left|\mathbf{k}^{\prime}\right|=2^{h^{*}},|\mathbf{p}| \leqslant 2^{h^{*}}$, and $l \in\{1,2\}$,

$$
\begin{aligned}
& \hat{S}_{2,0}^{h^{*}}\left(\mathbf{p}_{F}^{r}+\mathbf{k}^{\prime}\right)=\frac{\hat{g}_{r}^{\left(h^{*}\right)}\left(\mathbf{k}^{\prime}\right)}{Z_{h^{*}}}\left[1+O\left(e_{\mu, h^{*}}^{2}\right)\right] \\
& \hat{S}_{2,1 ; \mu}^{h^{*}}\left(\mathbf{k}^{\prime}+\mathbf{p}_{F}^{r}, \mathbf{p}\right) \\
& \quad=i e \hat{g}_{r}^{\left(h^{*}\right)}\left(\mathbf{k}^{\prime}+\mathbf{p}\right) \frac{Z_{h^{*}}^{(\mu)}}{\left(Z_{h^{*}}\right)^{2}}\left[\Gamma_{r}^{\mu}\left(\vec{k}^{\prime}\right)+O\left(e_{\mu, h^{*}}^{2}\right)\right] \hat{g}_{r}^{\left(h^{*}\right)}\left(\mathbf{k}^{\prime}\right)
\end{aligned}
$$

If we insert these equations into Eq. (8), we get

$$
\frac{Z_{h}^{(0)}}{Z_{h}}=\left[1+O\left(e_{\mu, h}^{2}\right)\right], \quad \frac{Z_{h}^{(i)}}{Z_{h} v_{h}}=\left[1+O\left(e_{\mu, h}^{2}\right)\right], \quad i \in\{1,2\},
$$

which means that the effective charges $e_{\mu, h}$ converge to a nontrivial fixed point $e_{\mu,-\infty}=e+O\left(e^{3}\right)$. Moreover, ${ }^{20}$ $e_{0,-\infty}=e_{1,-\infty}=e_{2,-\infty}$. By using Eq. (9) and by proceeding in a similar way, we also find that $v_{\mu, h}=O\left(e^{2} 2^{h}\right)$, i.e., the effective photon mass vanishes in the infrared (see Ref. 20).

The effective parameters $Z_{h}^{(\mu)}, Z_{h}$ diverge as $h \rightarrow-\infty$, while $v_{h}$ tends to the speed of light: indeed, the flow equations for $Z_{h}$ and $v_{h}$ are

$$
\begin{aligned}
\frac{Z_{h-1}}{Z_{h}} & =1+\frac{e^{2}}{12 \pi^{2}} \log 2+O\left[e^{2}\left(1-v_{h}\right)\right], \\
\frac{v_{h-1}}{v_{h}} & =1+\frac{2 e^{2}}{5 \pi^{2}} \log 2\left(1-v_{h}\right),
\end{aligned}
$$

up to corrections of order $O\left(e^{4}\right)$ and $O\left(2^{h}\right)$ (for the full form, see Ref. 20). These flow equations finally imply that the wave-function renormalization diverges in the infrared, while the effective Fermi velocity increases up to the speed of light, both approaching their limits with an anomalous power law:

$$
Z_{h} \sim 2^{-\eta h}, \quad 1-v_{h} \sim 2^{\tilde{\eta} h},
$$

with $\eta=\frac{e_{-\infty}^{2}}{12 \pi^{2}}+O\left(e_{-\infty}^{4}\right)$ and $\tilde{\eta}=\frac{2 e_{-\infty}^{2}}{5 \pi^{2}}+O\left(e_{-\infty}^{4}\right)$.

\section{THE EXPANSION FOR THE CONDUCTIVITY}

The above integration procedure leads to an expansion of the conductivity in terms of powers of $e_{\mu, h}$; such renormalized expansion is a resummation of the naive perturbative expansion in $e$. It must be stressed that there is a big difference between these two expansions: while the one in $e_{\mu, h}$ is order by order finite (with explicit bounds on the growth of the $n$ th-order contributions ${ }^{20}$ ), the naive one in $e$ is plagued by $O\left(\log ^{n} \omega\right)$ divergences at order $n$. Therefore, the truncation of the renormalized expansion is expected to give much more accurate predictions than the naive one.

The conductivity, according to Eqs. (10), (6), and (11), can be obtained from the terms in $\mathcal{V}^{(h)}\left(\sqrt{Z_{h}} \psi^{(\leqslant h)}, A^{(\leqslant h)}+\right.$ $J)$ that are quadratic in $J$. The lowest-order terms are the ones proportional to $v_{h}$ in Eq. (13) [that, however, do not contribute to the conductivity, due to Eq. (10)] and the ones in $\mathcal{R} \mathcal{V}^{(h)}$ obtained by contracting two operators $Z_{h_{1}}^{(\mu)} e \hat{\jmath}_{\mu, \mathbf{p}}^{\left(\leqslant h_{1}\right)} J_{\mu, \mathbf{p}}$ and $Z_{h_{2}}^{(\mu)} e \hat{J}_{\mu, \mathbf{p}}^{\left(\leqslant h_{2}\right)} J_{\mu, \mathbf{p}}$ among themselves; in order to contribute to $\mathcal{R} \mathcal{V}^{(h)}$ the scales $h_{1}, h_{2}$ should be both $\geqslant h$ and at least one of the two should be equal to $h$. Such contributions can be graphically depicted in terms as in Fig. 1 and give (defining $\left.\sigma_{0}=\frac{\pi}{2} \frac{e^{2}}{h}\right)$

$$
\begin{aligned}
\frac{\sigma_{i i}(i \omega)}{\sigma_{0}}= & \frac{16}{3 \sqrt{3}} \frac{1}{\omega} \sum_{h, h^{\prime} \leqslant 0}^{r= \pm} \int \frac{d k_{0}}{2 \pi} \int_{\mathcal{B}} \frac{d \vec{k}^{\prime}}{|\mathcal{B}|} \frac{\left(Z_{\vec{h}}^{(i)}\right)^{2}}{Z_{h} Z_{h^{\prime}}} \\
& \times \operatorname{Tr}\left(\Gamma_{r}^{i}\left(\vec{k}^{\prime}\right) g_{r}^{(h)}\left(\mathbf{k}^{\prime}\right) \Gamma_{r}^{i}\left(\vec{k}^{\prime}\right)\right. \\
& \left.\times\left\{g_{r}^{\left(h^{\prime}\right)}\left[\mathbf{k}^{\prime}+(\omega, \overrightarrow{0})\right]-g_{r}^{\left(h^{\prime}\right)}\left(\mathbf{k}^{\prime}\right)\right\}\right),
\end{aligned}
$$

where (i) $\mathcal{B}$ is the first Brillouin zone and $|\mathcal{B}|=8 \pi^{2} /(3 \sqrt{3})$ is its area; and (ii) $\bar{h}=\max \left\{h, h^{\prime}\right\}$. Note that the effective parameters $Z_{h}^{(i)}, Z^{h}, v_{h}$ entering Eq. (20) are all functions of $e$ : if we expanded them in $e$ we would recover infinitely many graphs of the naive perturbation theory, all plagued by logarithmic divergences. Note also that Eq. (20) is not

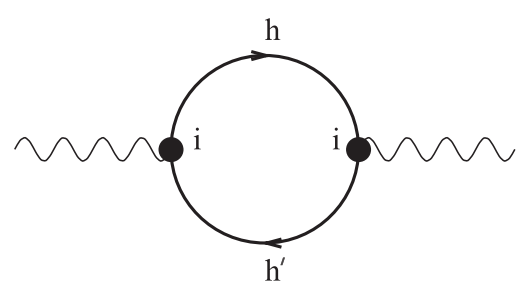

FIG. 1. The one-loop bubble diagram contributing to the longitudinal conductivity $\sigma_{i i}(\omega)$. The labels $h, h^{\prime}$ indicate the scale of the two loop propagators, which depend on the dressed Fermi velocities $v_{h}, v_{h^{\prime}}$ and on the effective wave function renormalizations $Z_{h}, Z_{h^{\prime}}$. A summation over $h, h^{\prime}$ is understood. The big dots correspond to dressed vertex functions $Z_{\bar{h}}^{(i)}$, with $\bar{h}=\max \left\{h, h^{\prime}\right\}$. The external momentum flowing in the wavy lines is $\mathbf{p}=(\omega, \overrightarrow{0})$. 
simply the "bubble graph" with the dressed propagator and vertices: e.g., if one thinks of the dressed propagator with momentum $\mathbf{k}$ as being obtained by resummations of the chain of self-energies, one has to take into account that the scales of the momenta flowing inside such self-energy subdiagrams are higher than the scale of $\mathbf{k}$, according to the rules of the exact $\mathrm{RG}$ (which avoid the problem of overlapping divergences and, correspondingly, the emergence of $n$ ! factors at higher orders).

The computation of Eq. (20) can be explicitly performed, by making use of Eq. (17) and by carefully exploiting symmetry cancellations that make the apparent logarithmic divergence of Eq. (20) finite (see the Appendix for details). The result is Eq. (2).

\section{CONCLUSIONS}

We performed an exact RG analysis of the conductivity in a lattice gauge model for graphene, in which not only the Coulomb interactions but also the retardation effects of the e.m. field are taken into account, thus avoiding some unphysical features of the model with static interactions. We find that the system behaves in qualitative agreement with the experimental data, in particular with the observed dramatic increase of the Fermi velocity and with the universality of the conductivity up to negligible power-law corrections $\left(\sim \omega^{2}\right)$ at low frequencies. The result is found by truncating an exact renormalized expansion that is order by order finite (i.e., it is not plagued by logarithmic divergences, unlike the perturbative expansion in the bare parameters) and by exploiting dramatic cancellations following from exact lattice gauge invariance.
It would be interesting, of course, to go beyond the one-loop analysis in the renormalized expansion, and check whether the conductivity is truly universal in the zero- frequency limit at all orders; the proof of universality of the optical conductivity for short-ranged interactions in Ref. 10 cannot be adapted to the Coulomb case and new ideas seem to be necessary. Moreover, our bounds at all orders in the renormalized expansion are performed in the Euclidean region (imaginary time) and the analytic extension to real frequencies is performed after the truncation. The use of imaginary time and frequencies in the collisionless regime $k_{B} T \ll \omega \ll \varepsilon$ does not miss any obvious relevant feature of the problem, but of course a more quantitative understanding of the analytical extension to real frequencies would be desirable. Finally, in deriving Eq. (2), we assumed that the values of the bare parameters are sufficiently close to the infrared fixed point. The extension to a larger range of parameters would require a microscopic justification that is quite difficult in view of the strength of interactions in graphene, but of course this is a caveat that applies to all the approaches based on expansions, resummations, and truncations.

\section{ACKNOWLEDGMENTS}

We acknowledge financial support from the ERC Starting Grant No. CoMBoS-239694. V.M. wishes to thank the Institute for Advanced Studies in Princeton where this work was completed.

\section{APPENDIX: COMPUTATION OF THE CONDUCTIVITY}

Using the definition of $g_{r}^{(h)}$ in Eq. (20), we can rewrite [shifting the momenta by $\vec{p}_{F}^{r}$ and neglecting, for consistency, terms of order $O\left(e^{4}\right)$, which should be combined with the two-loop contributions]

$$
\begin{aligned}
\frac{\sigma_{i i}(i \omega)}{\sigma_{0}}= & \frac{4}{\pi^{2}} \frac{1}{\omega} \sum_{h, h^{\prime} \leqslant 0}^{r= \pm} \int \frac{d k_{0}}{2 \pi} \int_{\mathcal{B}} d \vec{k}^{\prime} \frac{\left(Z_{\vec{h}}^{(i)}\right)^{2}}{Z_{h} Z_{h^{\prime}}} \frac{f_{h}\left(\left|\vec{k}^{\prime}\right|\right) f_{h^{\prime}}\left(\left|\vec{k}^{\prime}\right|\right)}{k_{0}^{2}+v_{h}^{2}\left|\Omega_{r}\left(\vec{k}^{\prime}\right)\right|^{2}}\left[\frac{-k_{0}\left(k_{0}+\omega\right)\left|a_{i, r}\left(\vec{k}^{\prime}\right)\right|^{2}+v_{h} v_{h^{\prime}} \operatorname{Re}\left[\Omega_{r}^{2}\left(\vec{k}^{\prime}\right) a_{i, r}^{2}\left(\vec{k}^{\prime}\right)\right]}{\left(k_{0}+\omega\right)^{2}+v_{h^{\prime}}^{2}\left|\Omega_{r}\left(\vec{k}^{\prime}\right)\right|^{2}}\right. \\
& \left.+\frac{k_{0}^{2}\left|a_{i, r}\left(\vec{k}^{\prime}\right)\right|^{2}-v_{h} v_{h^{\prime}} \operatorname{Re}\left[\Omega_{r}^{2}\left(\vec{k}^{\prime}\right) a_{i, r}^{2}\left(\vec{k}^{\prime}\right)\right]}{k_{0}^{2}+v_{h^{\prime}}^{2}\left|\Omega_{r}\left(\vec{k}^{\prime}\right)\right|^{2}}\right],
\end{aligned}
$$

where $\omega>0, \Omega_{r}\left(\vec{k}^{\prime}\right)=\Omega\left(\vec{k}^{\prime}+\vec{p}_{F}^{r}\right)$, and

$$
a_{i, r}\left(\vec{k}^{\prime}\right)=\frac{2}{3} \sum_{j=1}^{3}\left(\vec{\delta}_{j}\right)_{i} i e^{-i\left(\vec{k}^{\prime}+\vec{p}_{F}^{r}\right)\left(\vec{\delta}_{j}-\vec{\delta}_{1}\right)}
$$

Note that $\Omega_{r}\left(\vec{k}^{\prime}\right)=i k_{1}^{\prime}+r k_{2}^{\prime}+O\left(\left|\vec{k}^{\prime}\right|^{2}\right), a_{1, r}\left(\vec{k}^{\prime}\right)=i+O\left(\left|\vec{k}^{\prime}\right|\right)$, and $a_{2, r}\left(\vec{k}^{\prime}\right)=-r+O\left(\left|\vec{k}^{\prime}\right|\right)$. Note that the contributions from $r=+$ or $r=-$ are equal among each other, thanks to the symmetry under valley exchange. By performing the integral over $k_{0}$ using the residues' theorem, we find

$$
\frac{\sigma_{i i}(i \omega)}{\sigma_{0}}=\frac{4 \omega}{\pi^{2}} \int_{\mathcal{B}} d \vec{k}^{\prime} \sum_{h, h^{\prime} \leqslant 0} \frac{\left(Z_{\bar{h}}^{(i)}\right)^{2}}{Z_{h} Z_{h^{\prime}}} \frac{f_{h}\left(\left|\vec{k}^{\prime}\right|\right) f_{h^{\prime}}\left(\left|\vec{k}^{\prime}\right|\right)}{\left|\Omega_{+}\left(\vec{k}^{\prime}\right)\right|\left(v_{h}+v_{h^{\prime}}\right)} \frac{\left|a_{i,+}\left(\vec{k}^{\prime}\right)\right|^{2}-\left|\Omega_{+}\left(\vec{k}^{\prime}\right)\right|^{-2} \operatorname{Re}\left[\Omega_{+}^{2}\left(\vec{k}^{\prime}\right) a_{i,+}^{2}\left(\vec{k}^{\prime}\right)\right]}{\omega^{2}+\left(v_{h}+v_{h^{\prime}}\right)^{2}\left|\Omega_{+}\left(\vec{k}^{\prime}\right)\right|^{2}} .
$$

We can now perform analytical continuation to real frequencies $i \omega \rightarrow \omega+i 0^{+}$and, using the fact that $\frac{1}{x+i 0^{+}}=-i \pi \delta(x)+\mathrm{P} . \mathrm{V} \cdot \frac{1}{x}$ in the sense of distributions (here P.V. indicates Cauchy's principal value), we get 


$$
\begin{aligned}
\frac{\operatorname{Re} \sigma_{i i}\left(\omega+i 0^{+}\right)}{\sigma_{0}}= & \frac{2}{\pi} \int_{\mathcal{B}} d \vec{k}^{\prime} \sum_{h, h^{\prime} \leqslant 0} \frac{\left(Z_{\vec{h}}^{(i)}\right)^{2}}{Z_{h} Z_{h^{\prime}}} \frac{f_{h}\left(\left|\vec{k}^{\prime}\right|\right) f_{h^{\prime}}\left(\vec{k}^{\prime} \mid\right)}{\left|\Omega_{+}\left(\vec{k}^{\prime}\right)\right|\left(v_{h}+v_{h^{\prime}}\right)} \delta\left[\omega-\left(v_{h}+v_{h^{\prime}}\right)\left|\Omega_{+}\left(\vec{k}^{\prime}\right)\right|\right]\left\{\left|a_{i,+}\left(\vec{k}^{\prime}\right)\right|^{2}\right. \\
& \left.-\left|\Omega_{+}\left(\vec{k}^{\prime}\right)\right|^{-2} \operatorname{Re}\left[\Omega_{+}^{2}\left(\vec{k}^{\prime}\right) a_{i,+}^{2}\left(\vec{k}^{\prime}\right)\right]\right\} .
\end{aligned}
$$

A similar expression is valid for the imaginary part:

$$
\frac{\operatorname{Im} \sigma_{i i}\left(\omega+i 0^{+}\right)}{\sigma_{0}}=\frac{4 \omega}{\pi^{2}} \mathrm{P} . \mathrm{V} \cdot \int_{\mathcal{B}} d \vec{k}^{\prime} \sum_{h, h^{\prime} \leqslant 0} \frac{\left(Z_{\bar{h}}^{(i)}\right)^{2}}{Z_{h} Z_{h^{\prime}}} \frac{f_{h}\left(\left|\vec{k}^{\prime}\right|\right) f_{h^{\prime}}\left(\left|\vec{k}^{\prime}\right|\right)}{\left|\Omega_{+}\left(\vec{k}^{\prime}\right)\right|\left(v_{h}+v_{h^{\prime}}\right)} \frac{\left|a_{i,+}\left(\vec{k}^{\prime}\right)\right|^{2}-\left|\Omega_{+}\left(\vec{k}^{\prime}\right)\right|^{-2} \operatorname{Re}\left[\Omega_{+}^{2}\left(\vec{k}^{\prime}\right) a_{i,+}^{2}\left(\vec{k}^{\prime}\right)\right]}{\omega^{2}-\left(v_{h}+v_{h^{\prime}}\right)^{2}\left|\Omega_{+}\left(\vec{k}^{\prime}\right)\right|^{2}} .
$$

We start by analyzing Eq. (A3). Let $\rho, \theta$ be the polar coordinates of $\vec{k}^{\prime}$ and note that, for small $\rho$,

$$
\begin{aligned}
& \left|\Omega_{+}\left(\vec{k}^{\prime}\right)\right|=\rho\left[1+\rho b_{1}(\theta)+O\left(\rho^{2}\right)\right], \quad\left|a_{i,+}\left(\vec{k}^{\prime}\right)\right|^{2}=1+\rho b_{2}(\theta)+O\left(\rho^{2}\right), \\
& \frac{\operatorname{Re}\left[\Omega_{+}^{2}\left(\vec{k}^{\prime}\right) a_{i,+}^{2}\left(\vec{k}^{\prime}\right)\right]}{\left|\Omega_{+}\left(\vec{k}^{\prime}\right)\right|^{2}}=1-2 \cos ^{2} \theta+\rho b_{3}(\theta)+O\left(\rho^{2}\right),
\end{aligned}
$$

for suitable functions $b_{j}(\theta)$ that are odd in $\theta$. By using Eq. (A5) and the Ward identity, Eq. (17), we can rewrite Eq. (A3) as (neglecting higher-order corrections in $e^{2}$ )

$$
\begin{aligned}
\frac{\operatorname{Re} \sigma_{i i}\left(\omega+i 0^{+}\right)}{\sigma_{0}}= & \sum_{h, h^{\prime} \leqslant 0} \frac{Z_{\bar{h}}^{2}}{Z_{h} Z_{h^{\prime}}} \frac{4 v_{\bar{h}}^{2}}{\left.v_{h}+v_{h^{\prime}}\right)^{2}} \int_{0}^{2 \pi} \frac{d \theta}{2 \pi}\left[1-\frac{3 \omega}{v_{h}+v_{h^{\prime}}} b_{1}(\theta)+O\left(\omega^{2}\right)\right] f_{h}(\rho(\omega, \theta)) f_{h^{\prime}}(\rho(\omega, \theta)) \\
& \times\left\{2 \cos ^{2} \theta+\frac{\omega}{v_{h}+v_{h^{\prime}}}\left[b_{2}(\theta)-b_{3}(\theta)\right]+O\left(\omega^{2}\right)\right\},
\end{aligned}
$$

where $\rho(\omega, \theta)=\frac{\omega}{v_{h}+v_{h^{\prime}}}\left[1-\frac{\omega}{v_{h}+v_{h^{\prime}}} b_{1}(\theta)+O\left(\omega^{2}\right)\right]$, so that

$$
f_{h}(\rho(\omega, \theta))=f_{h}\left(\frac{\omega}{v_{h}+v_{h^{\prime}}}\right)-\frac{\omega^{2} \partial_{\rho} f_{h}\left(\frac{\omega}{v_{h}+v_{h^{\prime}}}\right)}{\left(v_{h}+v_{h^{\prime}}\right)^{2}} b_{1}(\theta)+\cdots .
$$

By the compact support properties of $f_{h}$, the integrand is nonvanishing only if $\left|h-h^{\prime}\right| \leqslant 1$, in which case

$$
E_{h, h^{\prime}}=\frac{Z_{\bar{h}}^{2}}{Z_{h} Z_{h^{\prime}}} \frac{4 v_{\bar{h}}^{2}}{\left(v_{h}+v_{h^{\prime}}\right)^{2}}-1
$$

is of order $O\left(e^{2}\right)$, uniformly in $h$. Therefore, using the fact that $b_{j}(-\theta)=-b_{j}(\theta)$, and integrating Eq. (A6) over $\theta$, we find

$$
\frac{\operatorname{Re} \sigma_{i i}\left(\omega+i 0^{+}\right)}{\sigma_{0}}=\sum_{h, h^{\prime} \leqslant 0}\left(1+E_{h, h^{\prime}}\right)\left[f_{h}\left(\frac{\omega}{v_{h}+v_{h^{\prime}}}\right) f_{h^{\prime}}\left(\frac{\omega}{v_{h}+v_{h^{\prime}}}\right)+\tilde{f}_{h, h^{\prime}}(\omega) F(\omega)\right],
$$

where $\tilde{f}_{h, h^{\prime}}(\omega)$ is a compact support function, bounded in absolute value by a constant independent of $h, h^{\prime}$ and with the same qualitative support properties as $f_{h}\left(\frac{\omega}{v_{h}+v_{h^{\prime}}}\right) f_{h^{\prime}}\left(\frac{\omega}{v_{h}+v_{h^{\prime}}}\right)$ [that is, $\tilde{f}_{h, h^{\prime}}(\omega)$ is different from zero only if $\left|h-h^{\prime}\right| \leqslant 1$ and if $c \gamma^{h} \leqslant \omega \leqslant C \gamma^{h}$ ]; $F(\omega)$ is bounded in absolute value by (const.) $\omega^{2}$. The right-hand side of Eq. (A8) is immediate to estimate and we finally get

$$
\frac{\operatorname{Re} \sigma_{i i}\left(\omega+i 0^{+}\right)}{\sigma_{0}}=1+O\left(\omega^{2}\right)
$$

up to terms bounded uniformly in $\omega$ by (const.) $e^{2}$, which should be neglected, for consistency, because the equation for the conductivity was obtained by truncating the exact RG expansion at one loop. This proves Eq. (2).

The imaginary part of the conductivity can be bounded in a similar way. We set $\varepsilon$ to be a small but finite fraction of the bandwidth (say, $\varepsilon=t / 10$ ) and we distinguish the contributions to the integral coming from the region $v_{0}\left|\Omega_{r}\left(\vec{k}^{\prime}\right)\right| \geqslant \varepsilon$ from those $v_{0}\left|\Omega_{r}\left(\vec{k}^{\prime}\right)\right| \leqslant \varepsilon$. The contributions from $v_{0}\left|\Omega_{r}\left(\vec{k}^{\prime}\right)\right| \geqslant \varepsilon$ are of the form $\omega$ times a nonsingular integral and they can be bounded by (const.) $\omega / \varepsilon$. The contributions from the region $v_{0}\left|\Omega_{r}\left(\vec{k}^{\prime}\right)\right| \leqslant \varepsilon$ can be written as

$$
\frac{8 \omega}{\pi} \sum_{h, h^{\prime} \leqslant 0} \frac{Z_{\bar{h}}^{2} v_{\bar{h}}^{2}}{Z_{h} Z_{h^{\prime}}} \text { P.V. } \int_{0}^{2 \pi} \frac{d \theta}{2 \pi} \int_{0}^{\varepsilon / v_{0}} d x\left[1-3 x b_{1}(\theta)+O\left(x^{2}\right)\right] \frac{f_{h}(\bar{\rho}(x, \theta)) f_{h^{\prime}}(\bar{\rho}(x, \theta))}{v_{h}+v_{h^{\prime}}} \frac{2 \cos ^{2} \theta+x\left[b_{2}(\theta)-b_{3}(\theta)\right]+O\left(x^{2}\right)}{\omega^{2}-\left(v_{h}+v_{h^{\prime}}\right)^{2} x^{2}},
$$


where we changed variable from $\vec{k}^{\prime}=(\rho \cos \theta, \rho \sin \theta)$ to $x=\left|\Omega_{+}\left(\vec{k}^{\prime}\right)\right|$ and $\theta$, so that $\bar{\rho}(x, \theta)=x\left[1-x b_{1}(\theta)+O\left(x^{2}\right)\right]$ and $f_{h}(\bar{\rho}(x, \theta))=f_{h}(x)-x^{2} \partial_{x} f_{h}(x) b_{1}(\theta)+\cdots$. Integration over $\theta$ yields

$$
\frac{2 \omega}{\pi} \sum_{h, h^{\prime} \leqslant 0}\left(1+E_{h, h^{\prime}}\right) \text { P.V. } \int_{0}^{\varepsilon\left(v_{h}+v_{h^{\prime}}\right) / v_{0}} d x \frac{1}{\omega^{2}-x^{2}}\left[f_{h}(x) f_{h^{\prime}}(x)+\tilde{f}_{h, h^{\prime}}(x) R(x)\right],
$$

where $\tilde{f}_{h, h^{\prime}}(x)$ is a compact support function with the same qualitative support properties as $f_{h}(x) f_{h^{\prime}}(x)$ and $R(x)$ is bounded in absolute value by (const.) $x^{2}$, while its derivative is bounded by (const.) $x$.

The term with the integrand proportional to $\tilde{f}_{h, h^{\prime}}(x) R(x)$ is the easiest to estimate and can be worked out as follows: we first distinguish the contributions from the integration region $|\omega-x| \geqslant \omega \delta$ from those $|\omega-x|<\omega \delta$, with $\delta$ a small but finite number. The contributions from $|\omega-x| \geqslant \omega \delta$ are easily bounded dimensionally by $O\left(\omega^{2}\right)$; the "singular ones" can be rewritten as

$$
\frac{1}{\pi} \sum_{h, h^{\prime} \leqslant 0}\left(1+E_{h, h^{\prime}}\right)\left[\tilde{f}_{h, h^{\prime}}(\omega) R(\omega) \text { P.V. } \int_{\omega(1-\delta)}^{\omega(1+\delta)} d x \frac{2 \omega}{\omega^{2}-x^{2}}+2 \omega \int_{\omega(1-\delta)}^{\omega(1+\delta)} d x \frac{\tilde{f}_{h, h^{\prime}}(x) R(x)-\tilde{f}_{h, h^{\prime}}(\omega) R(\omega)}{\omega^{2}-x^{2}}\right],
$$

which is equal to

$$
\frac{1}{\pi} \sum_{h, h^{\prime} \leqslant 0}\left(1+E_{h, h^{\prime}}\right)\left\{\tilde{f}_{h, h^{\prime}}(\omega) R(\omega) \log \frac{2+\delta}{2-\delta}-\int_{\omega(1-\delta)}^{\omega(1+\delta)} d x \frac{2 \omega}{x+\omega} \partial_{x}\left[\tilde{f}_{h, h^{\prime}}\left(x^{*}\right) R\left(x^{*}\right)\right]\right\},
$$

where $x^{*}$ is an interpolation point between $x$ and $\omega$. Equation (A13) is dimensionally bounded by $O\left(\omega^{2}\right)$, as desired.

We are left with the leading term in Eq. (A11), that is,

$$
\frac{2 \omega}{\pi} \sum_{h, h^{\prime} \leqslant 0}\left(1+E_{h, h^{\prime}}\right)\left[\text { P.V. } \int_{0}^{2 \varepsilon / v_{0}} d x \frac{f_{h}(x) f_{h^{\prime}}(x)}{\omega^{2}-x^{2}}+\int_{2 \varepsilon / v_{0}}^{\varepsilon\left(v_{h}+v_{h^{\prime}}\right) / v_{0}} d x \frac{f_{h}(x) f_{h^{\prime}}(x)}{\omega^{2}-x^{2}}\right] .
$$

The term corresponding to the second integral in square barckets is nonsingular and leads to a contribution to the conductivity bounded by $O(\omega)$. The first line is equal to

$$
\frac{1}{\pi} \text { P.V. } \int_{0}^{2 \varepsilon / v_{0}} d x \frac{2 \omega}{\omega^{2}-x^{2}}+\frac{2 \omega}{\pi} \sum_{h, h^{\prime} \leqslant 0} E_{h, h^{\prime}} \text { P.V. } \int_{0}^{2 \varepsilon / v_{0}} d x \frac{f_{h}(x) f_{h^{\prime}}(x)}{\omega^{2}-x^{2}},
$$

which can be further rewritten as

$$
\begin{aligned}
& \frac{1}{\pi} \log \frac{2 \varepsilon / v_{0}+\omega}{2 \varepsilon / v_{0}-\omega}+\frac{1}{\pi} \sum_{h, h^{\prime} \leqslant 0} E_{h, h^{\prime}} f_{h}(\omega) f_{h^{\prime}}(\omega) \text { P.V. } \int_{\omega(1-\delta)}^{\omega(1+\delta)} d x \frac{2 \omega}{\omega^{2}-x^{2}} \\
& \quad+\frac{2 \omega}{\pi} \sum_{h, h^{\prime} \leqslant 0} E_{h, h^{\prime}} \int_{\omega(1-\delta)}^{\omega(1+\delta)} d x \frac{f_{h}(x) f_{h^{\prime}}(x)-f_{h}(\omega) f_{h^{\prime}}(\omega)}{\omega^{2}-x^{2}}+\frac{2 \omega}{\pi} \sum_{h, h^{\prime} \leqslant 0} E_{h, h^{\prime}} \int_{0}^{2 \varepsilon / v_{0}} d x \frac{f_{h}(x) f_{h^{\prime}}(x)}{\omega^{2}-x^{2}} \chi(|\omega-x|>\omega \delta) .
\end{aligned}
$$

The second term in the first line is equal to

$$
\frac{1}{\pi} \sum_{h, h^{\prime} \leqslant 0} E_{h, h^{\prime}} f_{h}(\omega) f_{h^{\prime}}(\omega) \log [(2+\delta) /(2-\delta)],
$$

which is bounded by $O\left(e^{2}\right)$, uniformly in $\omega$. The first term in the second line is equal to

$$
-\frac{1}{\pi} \sum_{h, h^{\prime} \leqslant 0} E_{h, h^{\prime}} \int_{\omega(1-\delta)}^{\omega(1+\delta)} d x \frac{2 \omega}{\omega+x} \partial_{x}\left[f_{h}\left(x^{*}\right) f_{h^{\prime}}\left(x^{*}\right)\right]
$$

(here $x^{*}$ is an interpolation point between $x$ and $\omega$ ), which is bounded by $O\left(e^{2}\right)$, uniformly in $\omega$. Similarly, the second term in the second line of Eq. (A16) is bounded by $O\left(e^{2}\right)$, uniformly in $\omega$. Putting all together we find that the imaginary part of the conductivity is bounded by $O(\omega)$, up to corrections of order $O\left(e^{2}\right)$, which should be neglected, for consistency.

${ }^{1}$ A. K. Geim and K. S. Novoselov, Nature Mater. 6, 183 (2007).

${ }^{2}$ R. R. Nair, P. Blake, A. N. Grigorenko, K. S. Novoselov,

T. J. Booth, T. Stauber, N. M. R. Peres, and A. K. Geim,
Science 320, 1308 (2008); Z. Q. Li, E. A. Henriksen, Z. Jiang, Z. Hao, M. C. Martin, P. Kim, H. L. Stormer, and D. N. Basov, Nat. Phys. 4, 532 (2008). 
${ }^{3}$ T. Stauber, N. M. Peres, and A. K. Geim, Phys. Rev. B 78, 085432 (2008).

${ }^{4}$ A. W. W. Ludwig, M. P. A. Fisher, R. Shankar, and G. Grinstein, Phys. Rev. B 50, 7526 (1994).

${ }^{5}$ K. Ziegler, Phys. Rev. B 75, 233407 (2007)

${ }^{6}$ V. P. Gusynin, S. G. Sharapov, and J. P. Carbotte, Phys. Rev. Lett. 96, 256802 (2006).

${ }^{7}$ L. A. Falkovsky and A. A. Varlamov, Eur. Phys. J. B 56, 281 (2007).

${ }^{8}$ M. I. Katsnelson, Europhys. Lett. 84, 37001 (2008).

${ }^{9}$ D. C. Elias, R. V. Gorbachev, A. S. Mayorov, S. V. Morozov, A. A. Zhukov, P. Blake, L. A. Ponomarenko, I. V. Grigorieva, K. S. Novoselov, F. Guinea, and A. K. Geim, Nat. Phys. 7, 701 (2011).

${ }^{10}$ A. Giuliani, V. Mastropietro, and M. Porta, Phys. Rev. B 83, 195401 (2011); Comm. Math. Phys. (in press), e-print arXiv:1101.2169v1 [cond-mat.str-el].

${ }^{11}$ J. González, F. Guinea, and M. A. H. Vozmediano, Phys. Rev. B 59, R2474 (1999).
${ }^{12}$ E. G. Mishchenko, Phys. Rev. Lett. 98, 216801 (2007).

${ }^{13}$ I. F. Herbut, V. Juricic, and O. Vafek, Phys. Rev. Lett. 100, 046403 (2008).

${ }^{14}$ D. E. Sheehy and J. Schmalian, Phys. Rev. Lett. 99, 226803 (2007).

${ }^{15}$ E. G. Mishchenko, Europhys. Lett. 83, 17005 (2008).

${ }^{16}$ D. E. Sheehy and J. Schmalian, Phys. Rev. B 80, 193411 (2009).

${ }^{17}$ V. Juricic, O. Vafek, and I. F. Herbut, Phys. Rev. B 82, 235402 (2010); e-print arXiv:0809.0725 (unpublished).

${ }^{18}$ M. A. H. Vozmediano, Philos. Trans. R. Soc. London, Ser. A 369, 2625 (2011).

${ }^{19}$ J. Gonzalez, F. Guinea, and M. A. H. Vozmediano, Nucl. Phys. B 424, 595 (1994).

${ }^{20}$ A. Giuliani, V. Mastropietro, and M. Porta, Phys. Rev. B 82, 121418 (2010); Ann. Phys. 327, 461 (2012).

${ }^{21}$ K. Damle and S. Sachdev, Phys. Rev. B 56, 8714 (1997). 